\title{
Die Reputator-Funktion in der Unternehmertheorie
}

\author{
Rezension zu Daniel Gerbaulet (2016): Der Unternehmer als Reputator, \\ Tübingen: Mohr Siebeck
}

\section{Funktionen in der Unternehmertheorie}

"Was den Unternehmer von den übrigen Wirten unterscheidet, ist gerade, dass er sich in seinem Handeln nicht durch das bestimmen lässt, was war und ist, sondern allein durch das, was seiner Meinung nach sein wird. Er sieht, wie alle übrigen Menschen, die Vergangenheit und die Gegenwart; doch er sieht die Zukunft anders als sie. Den Antrieb zum Handeln gibt ihm seine, von den Meinungen seiner Mitbürger abweichende Erwartung über die Gestaltung der Zukunft. Dass er von der Zukunft eine andere Auffassung hat als die Mitmenschen, dass er die Produktionsmittel anders bewertet, als sie sie bewerten, scheint ihm eine Quelle von Gewinnen zu erschließen, die er nutzen will « (Mises 1940: 531).

Der echte Unternehmer versucht demnach auf eigenes Risiko geschäftliche Nischen zu entdecken und zu besetzen, die von anderen nicht erkannt oder wegen der Ungewissheit des Erfolges gemieden werden. Realisiert der Unternehmer dann ein für die betreffende Nische günstiges Szenarium, können zwar Konsumenten, Arbeitnehmer etc. davon profitieren, der erfolgreiche Unternehmer profitiert mitunter aber besonders stark. Im Ergebnis geht es allen besser, die Ungleichheit steigt jedoch. Daher wird dem Entdecker der Nische der unternehmerische Erfolg nicht gegönnt und als gesellschaftliche Leistung verklärt. Der sich kollektivistisch gebende Neider mag sich nicht eingestehen, dass der erfolgreiche Unternehmer im "Wettbewerb als Entdeckungsverfahren " (Hayek 1968) auch das Risiko des Misserfolgs trägt, was einen risikogerechten und daher hohen Unternehmerlohn rechtfertigt. Wohl auch deshalb wird in der >modernen betriebswirtschaftlichen Forschung die klassische Unternehmertheorie vernachlässigt.

Unbeirrt vom Zeitgeist und der vorherrschenden Forschungstrends widmet sich das vorliegende Buch von Gerbaulet der Unternehmertheorie und fügt den Bildern des Unternehmers als (1) Träger der Ungewissheit (Knight), (2) Innovator (Schumpeter), (3) Arbitrageur (Kirzer) und (4) Koordinator (Casson) das Bild des Unternehmers als (5) Reputator (Gerbaulet) hinzu (vgl. 170). ${ }^{1}$

* PD Dr. Andreas Haaker, Grundsatzabteilung des DGRV - Deutscher Genossenschaftsund Raiffeisenverband e.V., Privatdozent an der FernUniversität in Hagen und Initiator von Wert-Ideen.Berlin, Kontakt: DGRV, Pariser Platz 3, D-10117 Berlin, Tel.: +49(0)30-726220981, E-Mail: haaker@Wert-Ideen.Berlin, Forschungsschwerpunkte: Methodologische Grundlagen der technologischen Rechnungslegungsforschung, Accounting for Value Investors.

1 Alle Zitate stammen, wenn nicht anders ausgewiesen, aus Gerbaulet (2016). 


\section{Das Unternehmerbild des Reputators}

Ein Reputator stellt in Anlehnung an den Begriff des Innovators gleichsam den Förderer des strategischen Wettbewerbsvorteils der Reputation eines Unternehmens dar. Dass diese Funktion zumindest in nicht eigentümergeführten Unternehmen kein 'Selbstläufer sein muss, mag sich an der zwischenzeitig wohl für notwendig erachteten Verankerung des >Leitbilds des Ehrbaren Kaufmanns in der Präambel des Deutschen Corporate Governance Kodex zeigen. Der Kodex zielt vornehmlich auf angestellte Manager ab, die anders als Unternehmer nicht das Risiko tragen. Gerbaulet kritisiert den »managerhaften « Anstrich der Unternehmertheorie und möchte bei seiner Analyse zu den »Gründungsvätern « (Mütter hat er wohl keine entdecken können) der Unternehmertheorie zurückgehen (2). Zielsetzung soll es dabei sein, »den Unternehmer als Schlüsselelement der Theorie der Unternehmung [wieder] ins Zentrum der Betrachtung zu rücken « (1), wobei das (funktionale) Unternehmerbild um den Reputationsaspekt erweitert wird. »Wesenskern dieses neugeschaffenen Unternehmerbildes eines Reputators ist (...) der Aufbau und die Pflege der aus ehrbarer unternehmerischer Tätigkeit des Unternehmers entstehenden Reputation und deren unternehmerische Verwendung und Vermarktung « (3). Die Beschreibung dieses »Wesenskerns" assoziiert ein planvolles betriebswirtschaftliches Vorgehen. Hier stellen sich dem Rezensenten einige Fragen: Ent- und besteht Reputation nicht eher als eine Art unbeabsichtigter Konsequenz von absichtlichen Handlungen, die in der institutionellen Betrachtung Analogien zu Hayeks Konzept der spontanen Ordnung aufweist? Der Adam Smith 'sche Bäcker versorgt nicht nur die Kunden im Eigeninteresse mit Brot, sondern baut dabei als Nebeneffekt auch Reputation auf, die den Bäcker um die Ecke im Wettbewerb an- oder vertreibt. Ist dies und die simultane »Verwendung und Vermarktung " seiner Reputation nicht eher als ungeplante oder sogar unplanbare Konsequenz seines Erwerbsstrebens zu betrachten? Das Thema Reputation entzieht sich jedenfalls rechnerischen Kalkülen und muss sich als Frage des prinzipiengeleiteten und kooperativen Handelns erweisen. Daher ist es konsequent, dass Gerbaulet den ehrbaren Kaufmann als Leitbild des Unternehmers behandelt. Den ehrbaren Kaufmann interpretiert er als personelle Komponente der ReputatorFunktion des Unternehmers, der die Reputation zu instrumentalisieren gedenkt.

Auf das grundlegende Leitbild des ehrbaren Kaufmanns wird recht knapp eingegangen (vgl. 50-55). Dieses lässt sich wie folgt auf den Punkt bringen: Der ehrbare Kaufmann verzichtet auf opportunistisches Verhalten und lässt entsprechende "goldene Gelegenheiten " bewusst verstreichen (vgl. 53). Nach Gerbaulet muss diese intrinsische "Gutmenschen-Attitüde» ökonomisch gerüstet werden. Vergleicht aber der Unternehmer den kurzfristigen Opportunismus-Erfolg mit höheren langfristigen (diskontierten) Erfolgseinbußen, handelt er schlicht rational. Warum aber ein rationaler "Verzicht auf Opportunismus (...) in diesem Szenario mit ehrbarem Verhalten selbstverständlich wenig gemein « (53) haben soll, erscheint erklärungsbedürftig. Soll der ehrbare Kaufmann besser aus irgendwelchen gesinnungsethischen Bewegungen irrational und gleichzeitig opportunistisch handeln? Ist der Verzicht auf Opportunismus unehrenhaft, nur weil er rational 
begründet ist? Entscheidend sind hinsichtlich der ehrbaren Kaufmannseigenschaft nach Ansicht des Rezensenten letztlich nur die Fälle, in denen sich Opportunismus lohnen könnte. Doch kann in einer ungewissen Welt niemand mit Gewissheit sagen und schon gar nicht mittels objektiver Wahrscheinlichkeiten kalkulieren, ob am Ende nicht die negativen Konsequenzen überwiegen, da dem Opportunisten sein Verhalten in gleicher Münze heimgezahlt wird (>Tit for tat‘, 'Wie du mir, so ich dir!^). Prinzipiell ehrbares Verhalten kann daher als rationale Heuristik sevolutionär vorteilhaft sein und sich nach dem Trial-and-Error-Verfahren entwickelt haben (vgl. Haaker 2013). Gerbaulet stellt hierzu in Anlehnung an Albach aus betriebswirtschaftlicher Perspektive fest: »Ehrbares Verhalten erfolgt nicht zum Selbstzweck und aus Edelmut des Akteurs, sondern zunächst einmal zur betriebswirtschaftlichen Existenzsicherung und darüber hinausgehenden Generierung von Wettbewerbsvorteilen « (55).

Auch wenn damit die Unternehmensethik ausgeklammert bleiben kann, hätte an dieser Stelle der Bezug zur marktwirtschaftlichen Ordnung vertieft werden können. Wilhelm Röpke wies nachdrücklich auf »die Marktverbundenheit und Marktabhängigkeit des Unternehmers, die ihrerseits den Prozeß der Marktwirtschaft entscheidend beeinflussen « (Röpke 1947: 663), hin. In dieser trägt der ehrbare Kaufmann eine Vorbildfunktion und eine Diskursverantwortung für den Erhalt der hierzulande mit dem Adjektiv ssozial versehenen Marktwirtschaft. Dabei eckt der Unternehmer als Reputator mitunter am sozial-kollektivistischen Zeitgeist an, der das Adjektiv als Korrektiv zur Marktwirtschaft missversteht. Kurzum: notwendigerweise marktwirtschaftlich orientierte Reputation als ehrbarer Kaufmann könnte gleichsam zum Wettbewerbsnachteil in einem sich als ssozial verstehenden Umfeld werden, worauf der Verfasser nicht näher eingeht und nur von einer »Disziplinierung zu ehrbarem Verhalten durch Markt und öffentliche Meinung " (55) ausgeht. Röpke sprach demgegenüber von einem notwendigen »Glauben, der ihm [d.h.: dem Unternehmer, A.H.] mehr als das gute Gewissen, der ihm den Elan einer im innersten verankerten Überzeugung von seiner sozialen Funktion und damit die Kraft gibt, den stürmischen kollektivistischen Tendenzen unserer Zeit zu widerstehen " (Röpke 1947: 668). Betriebswirtschaftlich und im Lichte der reputationsfördernden öffentlichen Meinung kann das Widerstandsvermögen des ehrbaren Kaufmanns im Gegensatz zum Opportunismus nachteilig sein.

\section{Die Reputator-Funktion aus institutionsökonomischer Sicht}

Auf dem Fundament der breit diskutierten Denkschule der Neuen Institutionenökonomik (vgl. 56-114) stützt sich das Reputator-Konzept zum einen auf die personale Säule des ehrbaren Kaufmanns und zum anderen auf die funktionale Säule der Reputation (vgl. 138). Bei der Reputation handelt es sich um eine strategische Wettbewerbsvorteile generierende Ressource, die der Unternehmer bestmöglich zu operationalisieren hat (vgl. 136). Gerbaulet bettet seine Reputator-Funktion in die institutionelle Sichtweise von Dieter Schneider ein, wobei sie als eine institutio- 
nen-verbessernde Funktion zu verstehen sein soll. Zu verbessern sind folgende der Schneider'schen Unternehmerfunktionen (vgl. 147):

- die institutionen-erhaltende Funktion nach innen, zu der die Koordinationsund Innovationfunktion gerechnet werden,

- die institutionen-erhaltende Funktion nach außen, zu der die Arbitragefunktion zählt, sowie

- die institutionen-begründende Funktion, welche die Übernahme von Einkommensunsicherheiten enthält.

Dabei soll die Reputator-Funktion »eine Art begleitende Handlungsmaxime " darstellen (161), deren Kernaspekte zum Ende der Schrift wie folgt verdichtet werden:

"Der Aufbau und die Pflege der aus ehrbarer unternehmerischer Tätigkeit entstehenden Reputation und im besonderen deren aktive unternehmerische Vermarktung determinieren und kennzeichnen den als Reputator handelnden Unternehmer. Der Abbau von Unsicherheiten, Informationsasymmetrien und Transaktionskosten, die Förderung von Kooperationen sowie die damit einhergehende Erlangung von Wettbewerbsvorteilen folgen dem Ziel einer Institutionen-verbessernden Unternehmerfunktion" (168).

Insgesamt gelingt es dem Verfasser jedenfalls den »Unternehmer als Schlüsselelement der Theorie der Unternehmung ins Zentrum der Betrachtung zu rücken « (1) und der von Dieter Schneider herausgestellten Bedeutung der Funktionen des Unternehmers für die betriebswirtschaftliche Theorie der Unternehmung gerecht zu werden. Als Denkrichtungen haben der methodologische Individualismus sowie die subjektive und funktionale Wertlehre erkennbare Spuren in der Arbeit hinterlassen. Der Unternehmer wird als individuell handelndes Subjekt betrachtet, welches nach seinen Wertvorstellungen Funktionen erfüllt. Der wissenschaftliche Wert der Arbeit besteht insbesondere darin, dass nicht nur eine Definition, sondern eine Explikation (theoriegeleitete Begriffserweiterung) des Unternehmerbegriffs vorgenommen wird. Der funktionale Unternehmerbegriff wird durch die Erweiterung der Reputator-Funktion für die Arbeit an bestimmten theoretischen Problemen fruchtbar gemacht. Ein hinsichtlich der Reputationsfunktion explizierter Unternehmerbegriff stellt also ein »besseres intellektuelles Werkzeug zur Bewältigung der gestellten Probleme« (Radnitzky 1989: 74f.) dar. Die Arbeit ist daher allen zu empfehlen, die sich tiefgründiger mit der Unternehmerfunktion beschäftigen möchten, um theoretische und praktische Probleme zu lösen. Sie liefert aufgrund der zahlreichen Verweise zudem einen guten Einstieg für ein Studium der historischen Originalquellen, die auch der nicht auf Linie von Gerbaulet liegenden positivistischen »Entrepreneurship-Forschung " (Hering 2016: V) von Nutzen sein dürfte.

\section{Literaturverzeichnis}

Gerbaulet, D. (2016): Der Unternehmer als Reputator, Tübingen: Mohr Siebeck.

Haaker, A. (2013): Nun sag, Homo oeconomicus, wie hast du's mit der Moral? Bestandsaufnahme und Grundgedanken zur 'moralisch-evolutionären` Fortentwicklung des ökonomi- 
schen Menschenbildes, in: Zeitschrift für Wirtschafts- und Unternehmensethik, Jg. 14/H. 2, 157-177.

Hayek, F. A. von (1968): Der Wettbewerb als Entdeckungsverfahren, in: Internationales Institut `Österreichische Schule der Nationalökonomie< (Hrsg.): Die Österreichische Schule der Nationalökonomie, Texte - Band II, Wien: Manz'sche Verlags- und Universitätsbuchhandlung, 119-137.

Hering, T. (2016): Geleitwort, in: Gerbaulet (2016), V-VI.

Mises, L. von (1940): Nationalökonomie, Genf: Editions Union.

Radnitzky, G. (1989): Explikation, in: Seiffert, H./Radnitzky, G. (Hrsg.): Handlexikon zur Wissenschaftstheorie, München: dtv, 73-80.

Röpke, W. (1947): Die Stellung des Unternehmers auf dem Markte, in: Schweizer Monatshefte, Jg. 26/H. 11, 663-674.

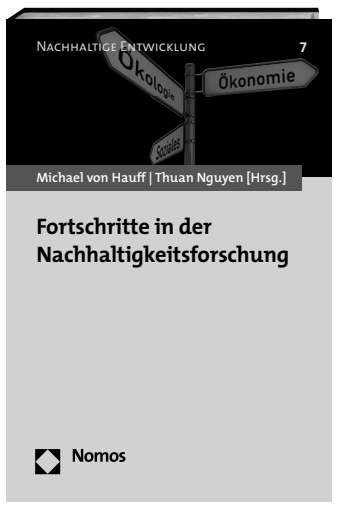

\section{Fortschritte in der Nachhaltigkeitsforschung}

Herausgegeben von Prof. em. Dr. Michael von Hauff und Thuan Nguyen, M.A.

2018, 350 S., brosch., 64,- $€$

ISBN 978-3-8487-4501-2

eISBN 978-3-8452-8745-4

(Nachhaltige Entwicklung, Bd. 7)

nomos-shop.de/30578

Nachhaltige Entwicklung findet an Hochschulen zunehmende Aufmerksamkeit. Hochschulen haben noch ein großes Potenzial, ihre erfolgreichen Aktivitäten im Rahmen nachhaltiger Entwicklung stärker zu präsentieren, aber auch in die Gesellschaft hinein zu tragen. Der vorliegende Sammelband leistet einen Beitrag dazu, indem er die Fortschritte und Herausforderungen der Nachhaltigkeitsforschung an der Technischen Universität Kaiserslautern in den verschiedenen Forschungsdisziplinen aufzeigt. Beeindruckend ist dabei die Verschiedenartigkeit der Forschungsprojekte in den einzelnen Disziplinen. Das Forschungsfeld nachhaltige Entwicklung erfordert auf der einen Seite interdisziplinäre Zusammenarbeit und bietet auf der anderen Seite ein großes Kooperationspotential.

స्ञाIIIIII Nomos Unser Wissenschaftsprogramm ist auch online verfügbar unter: હelibrary www.nomos-elibrary.de

Portofreie Buch-Bestellungen unter www.nomos-shop.de Alle Preise inkl. Mehrwertsteuer 